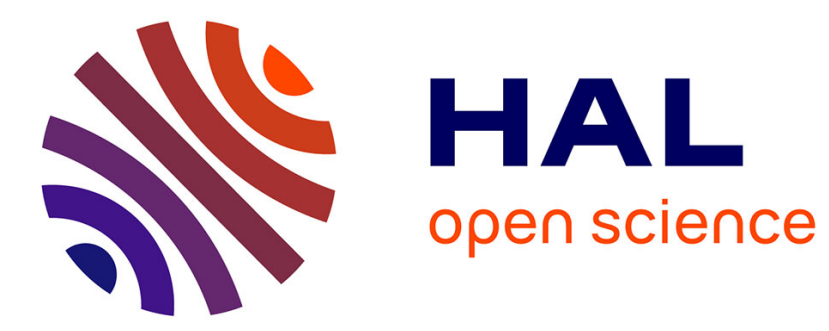

\title{
Les gisements préhistoriques de Belcayre (Dordogne)
}

Franck Delage

\section{To cite this version:}

Franck Delage. Les gisements préhistoriques de Belcayre (Dordogne). Gallia - Fouilles et monuments archéologiques en France métropolitaine, 1949, 7 (1), pp.3-21. 10.3406/galia.1949.2115 . hal01920783

\section{HAL Id: hal-01920783 \\ https://hal.science/hal-01920783}

Submitted on 27 Feb 2020

HAL is a multi-disciplinary open access archive for the deposit and dissemination of scientific research documents, whether they are published or not. The documents may come from teaching and research institutions in France or abroad, or from public or private research centers.
L'archive ouverte pluridisciplinaire HAL, est destinée au dépôt et à la diffusion de documents scientifiques de niveau recherche, publiés ou non, émanant des établissements d'enseignement et de recherche français ou étrangers, des laboratoires publics ou privés.

\section{(이) $\$$}

Distributed under a Creative Commons Attribution - NonCommercial - NoDerivatives $\mid 4.0$ 


\title{
I.ES GISEMENTS PREHISTORIQUES DE BELCAYRE
}

\author{
(Dorrlogme)
}

pau M. Francki Des.urie

Les gisements próhistoriques dr Belrayre sont situes sur le territoire de la rommune de Saint-líon-sur-l'ézère. tont auprès de la limito de relle de Thonar. Its se homrent dans un dos sites les plus charmants de

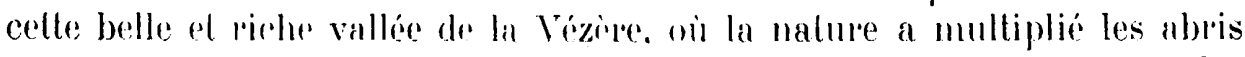
sous roche. I ring cents mictres en aval du petil bourg de? Thonar. des falaises abruptes dominent a pie la rive droite de la Vérover dans le coté concave d'un miandere oì le flot a rongé les bases des rochers. Ine. saillie itroite porte le pelit rhitean de Belcarre qui y loge loul juste sa lour ronde el son corps de logis, pittoresqur ivocation du $\mathrm{xv}^{\mathrm{e}}$ siecle Apres avoir baigne le pied de la falaise. la rivière. rejetée a l'opposé solon la loi du balancemenl des rives concaves el convexis. el s'orientant rers Sergeace sierarte rapidemenl el libère une zone de rochers plus bas. qui s'óloignenl progressivemenl des eaux. Cette zone montre non plus me abrupte falaise. mais des pans de rochers imrgulirmment espacés el des talus d'aboulis broussailleux.

Pour l'ensemble de relle zone. nous pourons indiguer l'existence de quatre gisements palédithiques. qui s'echelonment d'amont en aval dans l'ordre suivant (fig. 1): 1" au pied du rocher'. prestgur all-dessous du manoir. un petil gisement magdalénien. que nous dínommerons lo

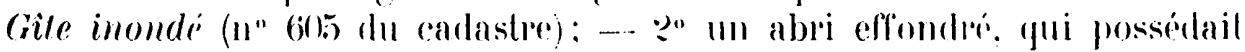
trois niveaux superpossis, moustérien. aurignacien. magdalónien: labri

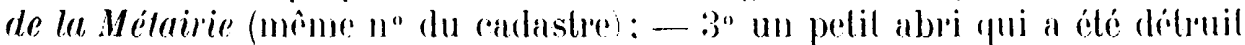
par une tres ancienne "arliere el par l'iblablissement d'un petil rhemin qui passe au-dessus de la métairie: - . 'o dans une zone d'éboulis. cent rinquante metres plus loin 'n aval. un gisement aurignareien. dil tbri de Ia gracure dlu Remue (nº 586 du cadastre)?

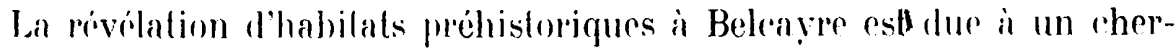
chenr souvent riti dans les monographies des stations do la Vézere. A.

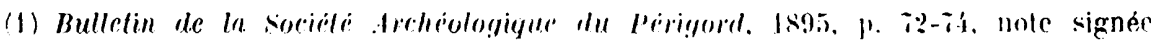

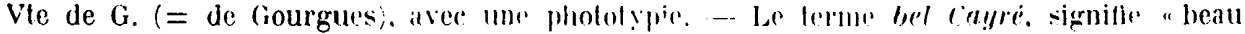
rocher ", "fler rocher". - St-Léon-sur-Vèrer fait partir dul ranton de Montignale, arrondissement de Sarlat.

(2) Tous ces gisements lont partir du domaine di. Brleayre. qui appartenait, it lepoque de nos fouilles, i . .1. Borias. - Le produil de mos lravaux dans les sites $2^{\circ}$ ef $t^{\circ}$ a eté remis par nous all Musér Sational des Eyzies. 
Reverdit, commis de culture des Tabacs à Montignac. Vers 1872-1874, Reverdit ramassa près des rochers de Belcayre ${ }^{1}$, une grande quantité de silex, mais sans faire de fouilles, sans mettre la pioche dans les talus pour chercher des couches. Il a noté la présence à Belcayre-Bas d'un reste de brèche adhérent au rocher, à une hauteur de plus d'un mètre par rapport au niveau du sol actuel, avec des silex empâtés dans cette brèche. Continuant ses récoltes en aval jusqu'à La Rochette, il eut la la conviction que tous les gisements "ne devaient former qu'une seule êt même station" sur un kilomètre de longueur et deux à trois cents

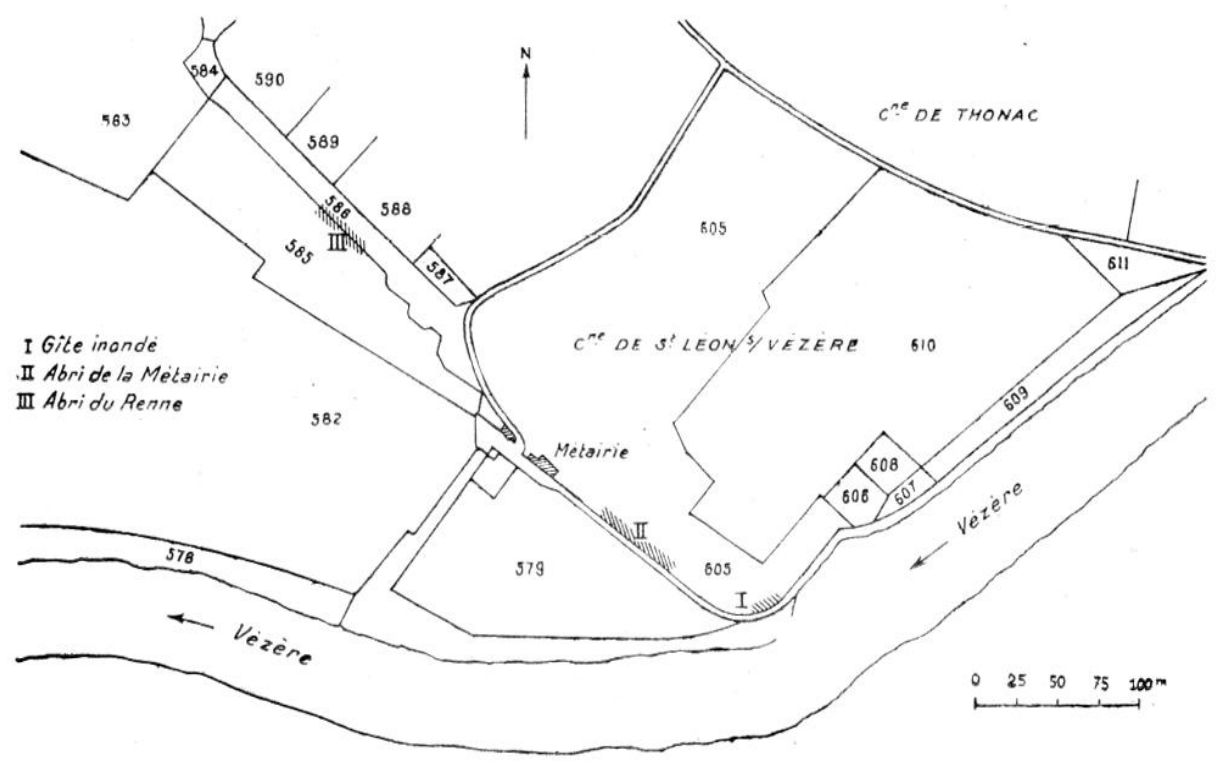

Fic. 1. - Situation des gisements de Belcayre sur le plan cadastral.

mètres de largeur moyenne ${ }^{2}$. Il ne s'efforça donc pas de différencier Belcayre de La Rochette. Dans ret ensemble, il signale essentiellement du Moustérien et du Solutréen: on ignorait alors l'Aurignacien.

Les parties les plus importantes des récoltes de Reverdit ayant passé dans la collection A. Sturge, qui est elle-même entrée au British Museum, nous avons pu savoir par les obligeants Conservateurs du Department of Antiquities, que quelques objets étiquetés Belcayre-Bas appartiennent au Solutréen I; ce sont une vingtaine de lames en forme de feuilles, généralement plutôt grossières, sauf une ou deux assez bonnes pour être appelées solulréennes. De plus, an Musée dul Périgord, il se trouve, sous l'étiquette Belcayre-Bas, deux fragments de "feuilles de laurier" et un

(1) Reverdit dénomma ces rochers Belcayre-Bas, pour les distinguer du plateau dit par lui Belcayre-Hlaut. Voir notre note sur Aurignacien du plateau de Belcayre, Congres prêhist. de France, XI session (Perigueux), 1934; p. 379-387, 31 1ig.

(2) Bull. de la Soc. des Sc. Nat. de Toulouse, VIII, 1873, pp. 34-35; Bull. de la soc. Arch. du Périgord, i, 18:8, p. 392-395. 
tronçon de "flèche à cran "1. On est donc porté à accepter l'existence du Solutréen à Belcayre-Bas; mais il est impossible, du moins pour le moment, d'indiquer où ce niveau était placé; nous n'avons, quant à nous, rien recueilli qui s'y rapporte. D'ailleurs, il ne faut pas oublier que Reverdit - selon ses propres termes - avait "récolté tous les silex de la collection au milieu des champs cultivés... sauf rares exceptions" ". Il est même probable qu'il a mis sous la même étiquette Belcayre et La Rochette.

Une fouille qui attaqua surtout la couche supérieure du gisement que nous avons dénommé Abri de la Métairie a été effectuée pendant quelques jours, aux environs de 1900, par l'abbé Landesque. Ce géologuepréhistorien n'a malheureusement laissé aucune relation, aucune note concernant cette fouille. Nous la connaissons seulement par les souvenirs de quelques personnes du voisinage ${ }^{3}$.

\section{I. - LE "GITE INONDE "}

Ce gite se trouve au tournant du rocher le long duquel passe l'étroit chemin qui remonte de la rivière ver's le château de Belcayre. Il est à une vingtaine de mètres des basses eaux. Les fortes crues - qui ne sont pas rares - ont fréquemment atteint et noyé ce gîte; une plaque fixée à la falaise à cet endroit-là, à 2 mètres au-dessus du chemin, signale la grande crue de 1876. Le long du rocher, à partir de la Vézère, le sol est sableux; mais, près du tournant, une petite butte de terre couvre le pied de la falaise.

C'est dans cette butte, à la faible profondeur de 10 à 15 centimètres, que MM. Borias ont recueilli, vers 1921-1922, un certain nombre de silex, qui, loin de paraître in situ, semblaient bien plutôt avoir été déposés là par le mouvement de l'eau. On peut supposer qu'il y a eu un petit gisement à une très faible distance en amont, le long de la roche, que ce gisement a été détruit par les crues, et que des reliquats ont été portés par l'eau jusqu'à la butte où ils se sont arrêtés. L'espace qu'ils occupaient ne dépasse pas $1 \mathrm{~m} .70$ de long sur 1 mètre de large.

Les silex recueillis sont des grattoirs, des burins et des lames, qui

(1) Catalogue de la collection prêhistorique, par M. FÉAcx, (1905), nos 4264, - 5, - 6. Mais M. FÉAux (lettre personnelle du 28-XI-1922), avant trouvé ces objets dans la collection Hardy, n'a pu être certain que la provenance fût Belcavre plutòt que La Rochette, Reverdit parlant de ces deux sites comme devant "former une mème station". A La Rochette, Elie Pevrony a signalé, avec un moustérien de tradition acheuléenne, un aurignacien moyen et un périgordien caractérisé, quelques fragments de feuilles de laurier et de pointes a face plane.

(2) Bulletins précités (p. 4, n. 2), respectivement p. 35 et 394 . - Reverdit signale en outre, dans ses récoltes de Belcayre-Bas, une hachette.polie en grès et une hachetteciseau en grès également.

(3) V. notre notice biographique L'Abbe Landesque, 1838-1905 (Bull. Soc. Arch. du Perigord, LVI, 1939, 4e fasc.). Les recherches faites par Landesque à Belcayre sont a ajouter à celles que cette notice a signalées. 
nous onl paru magdaleniens; il y arail rol oulere detix fragments de bois de renne portant des lareses dre travail. Sous ! avons remarqué aussi

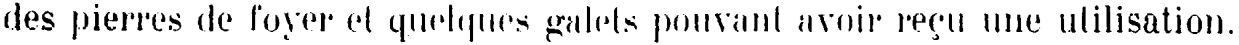

\section{1. - L.ABRI IIE I.I IIETAIRIE}

A 150 meles en aral du Gite inomde. loul auprès du rarrefour formé par un chemin par oi l'on descend du plateau à la mélairie, el par un autre chemin longeant vers l'amonl le pied des rochers, on voit un reste de falaise. long d'une dizaine de metres, dont la base est en partie dénudée. Ce pan de falaise forme un abri peu profond; il est ensuite lrès dégradé el ne présente plus aucun surplonb; il esl an quelque sorte fragmenti el se derobre en majerne parlie sous un lalus d'éboulis et dr leme. Au pird du lalus, fre rhemin latimal rst a deux mètres andessus de prís qui représentent le niveau moven de la vallée (fig. 2). Il n'y a pas de sédiments fluviatiles, mais senlement des apports par ruissellement du haul du platean supérieur. Le chemin, qui a éli construil vers 1885. recourre aussi le bas du lalus. ol par conséquent la partie inférieure dlu gisement, qui devail aller jusqu’a la prairie. La plate-forme

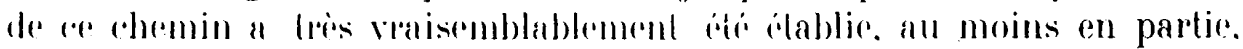
aver des lemes el des piprrailles prises dans le gisement. el plutöt dans le haut du gisement, c'est-i-dire lí oil nous arons découvert des couches aurignacienne el magdalénimno: il ast lros probable que les cantonniers n'ont pas tourhe aux courhes les plus profondes. Dans cette partie du gisement. (dans le sens nor(-sudli. pour commailre l'étendue complète des couches archéologiques, il eût fallu difoncer le chemin - ce qui citait impossible. Nous avons pu éludier dans ae gîle trois niveaur arrefrelogiques que nous décrirons en partant du bas.

\section{A) Virrern monstérien.}

Ce niveau a eté fouillí par nous entre le rhemin et la base dénudée de la falaise, al rarrefour indiquí plus haul. La partie postérieure butail contre le rocher. Nous avons décril précédemment le Moustérien de Belcayre '. on étudianl on détail les nucléi: les routeaux (souvent a dos). les éclats lriangulaires. les scies. les cisenux. les racloirs, les pièces à museau. les roches. les pirress à usages multiples. les éclats quadrangulaires, etc... Nous ne revicudrons pas sur ces remarques morphologiques. al nous nous bornerons ici i reprendre l'essentiel et les conclusions: d'abord, une reptaine lendance a rréer le grattoir à tête arquée, le grattoir caréné, et le burin "; puis. la fréquence de la retouche abrupte, très

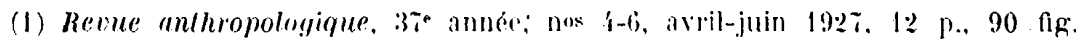

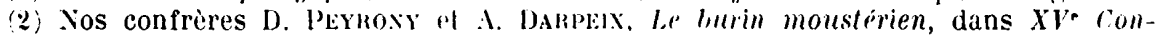
grès International d'anthropologie, 19:30, 1. I du lirage a pall... - el: Quelques obserrations sur le inoustérien du gisemenl Sandongne à Tabaterie, deurième note sur le burin 

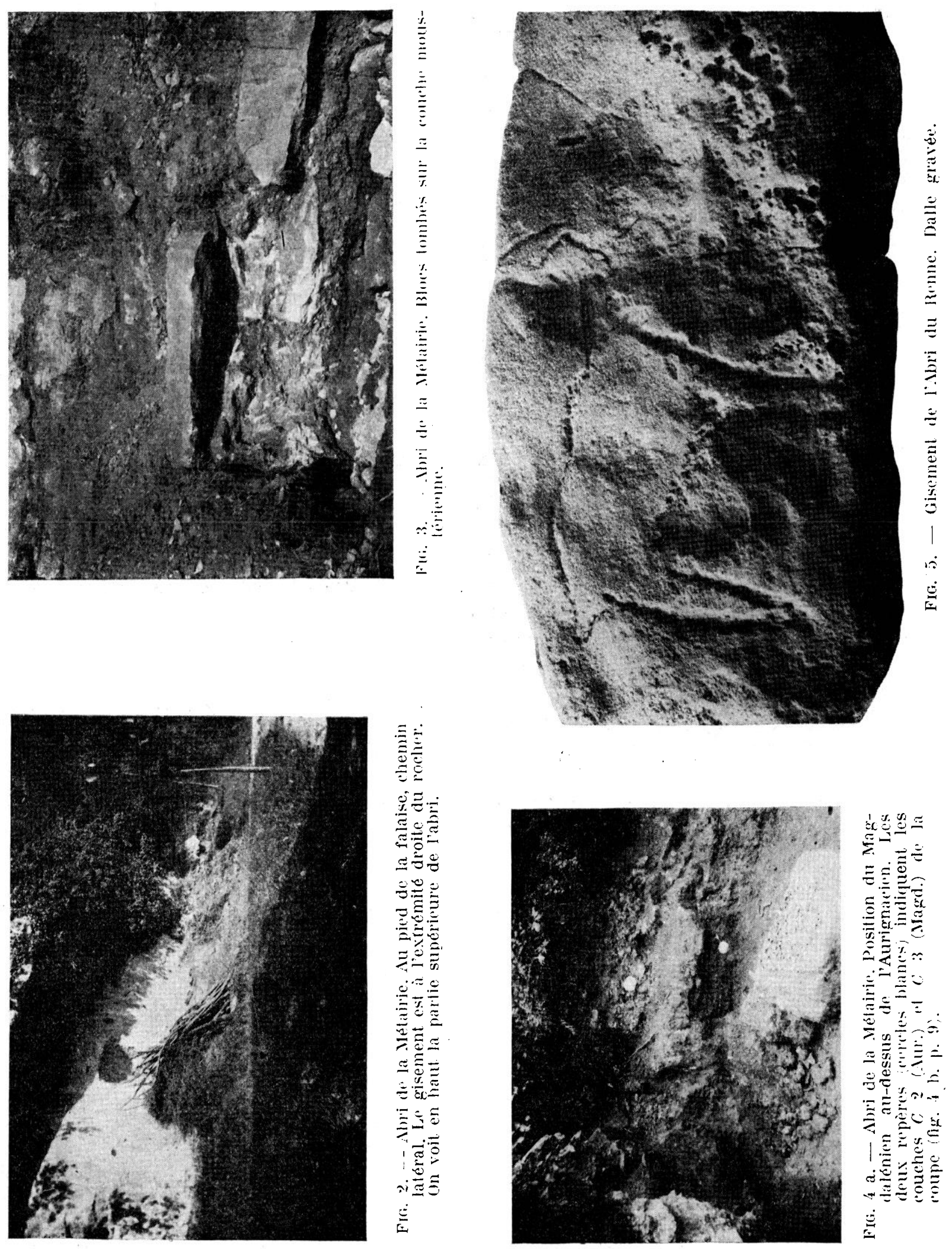
différente de la retouche proprement moustérienne; et enfin, la présence d'un coup-de-poing.

Cettc couche, qui élail incluse el comme scellée entre deux éboulis formés de blocs et de sortes de dalles provenant de l'effondrement de la corniche de l'abri, était nettement isolée du reste du gisement (fig. 3): pas d'autre couche au-dessus, à cause du déblaiement du pied de la falaise, ni au-dessous, comme un sondage nous l'a prouvé; donc, pas de point de repère local. Mais la comparaison de notre récolte avec ce qu'ont donné d'autres gisements moustériens du Périgord nous a permis de conclure ainsi: le moustérien de Belcayre s'apparente au moustérien de tradition acheuléenne, et répond plutôt à la dernière phase de cette tradition; il appartient au moustérien final et semble représenter les préludes de la première industrie aurignacienne ${ }^{1}$.

Aux objets publiés en 1927, nous ajoutons ici un léger supplément: des pièces à terminaison transversale formant un ciseau dégagé par des retouches symétriques à droite et à gauche, étranglant la pièce; des éclats minces à bords plus ou moins retouchés, dont un a l'aspect d'une fléchette; des prototypes de grattoirs sur éclats peu épais, avec retourhes, dentelures et écaillures symétriques; - un couteau-ciseau, bord droit assez fortement retouché, coche en dessous; - une pièce à usages multiples, racloir-pointe-coche; - une pointeperçoir, côté droit fortement retouché, cùté gauche très éraillé, pointe bien dégagée; - une pointe (petit museau): - et quelques prototypes carénés.

\section{B) Niveau aurignacien.}

Le reste du gisement s'est montré avec l'aspect suivant:

$1^{\circ}$ en face de la partie de falaise la mieux conservée, à toucher le carrefour des deux chemins ruraux, et un peu en contre-bas du sol rocheux déclive, une couche moustérienne, qu'aucun autre niveau archéologique ne surmontait. On peut supposer qu'il n'en était pas ainsi anciennement et qu'il $\mathrm{y}$ a eu déblaiement et expulsion d'un niveau supérieur quand fut édifié (époque gallo-romaine? haut moyen âge?) un habitat construit avec des poutres prenant appui sur des trous ou

moustérien, dans $X I^{\circ}$ Congrès préhistor. de France, 1934, p. 4 du tirage à part) ont émis l'opinion que les burins trouvés par nous proviennent d'un niveau aurignacien sus-jacent, et que, dans cette fouille, les formes aurignaciennes sont mêlées aux formes moustériennes. Nous persistons à croire que les burins quc nous avons signalés sont vraiment du niveau moustérien; il y avait, sur ce niveau, un barrage très net et que nous pour.rions dire imperméable.

(1) Il faut penser surtout à la couche $\mathrm{H}$ du Moustier (D. PeYrony, Le Moustier, Rev. Anthrop., 1930; tirage dे part p. 27, 47, 50). - CP. F. DelaGe, L'Abri des Merveilles,

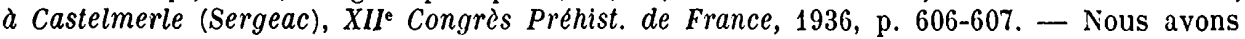
dit plus haut que Reverdit avait fusionné Belcayre et La Rochette; de fait, La Rochette a aussi du Moustérien et de tradition acheuléenne; toutefois, d'après les remarques de E. Peyrony (AFAS, Congrès de Bruxelles, 1939, p. 326) les coups-de-poing sont plus nombreux qu'd Belcayre, et, de plus, La Rochette avait du Moustérien typique. 
encoches creusés dans la falaise. On peut supposer aussi que des éboulements du surplomb de l'abri ont forré les Moustériens à quitter la place.

$2^{n}$ à la droite de celle zone, dans un espace de 5 mètres de long, vient un niveau anrignacien qui bulle conlre la partie supérieure d'ébou. lis situés à peine plus haut que les blocs qui recouvrent le moustérien. sement.

- Sur cette couche, un niveau magdalénien très net complète le gi-

La coupe de cette deuxième zone, par une tranchée perpendiculaire au rocher, présente de haut en bas (fig. $4 a$ et $b$ ): $a$ ) petit éboulis: 0,10;

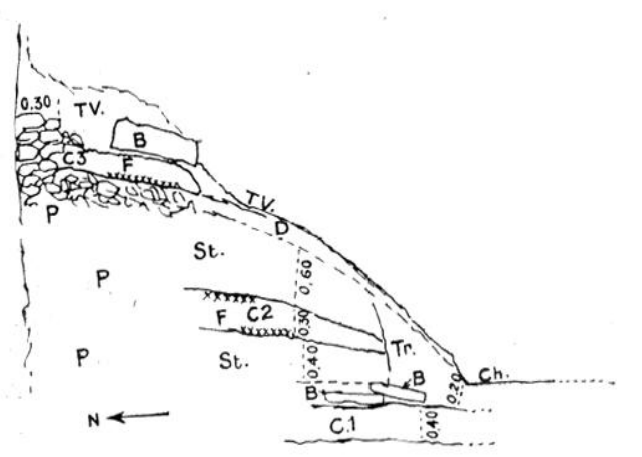

Fig. 4 b. - Abri de la Métairie. Coupe. (V. fig. 4 a.)

$\mathrm{TV}=$ Terre végétale.

$\mathrm{TR}=$ Terre remuée.

$\mathrm{D}=$ Déblais et relicta antérieurs.

$\mathrm{Ch}=$ Chemin latéral aux rochers.

$\mathrm{P}=$ Pierrailles et blocs.

St. $=$ Zone stérile.

$\mathrm{B}=$ Blocs.

$\mathrm{C} 1$ = Couche moustérienne.

$\mathrm{C} 2$ = Couche aurignacienne.

C3 = Couche magdalénienne.

$\mathrm{F}=$ Fover.

$-b$ ) éboulis à éléments plus forts, blocs: 0,$30 ;-c)$ couche magdalénienne: 0,$20 ;-d)$ éboulis intermédiaire: 0,25 ; $-e$ ) partie supérieure de la couche aurignacienne: $0,15 ;-f)$ éboulis séparatif: 0,20 ; - g) couche aurignacienne : 0,30 .

Des terres de déblais, qu'on assure provenir des recherches de l'abbé Landesque, courraient une partie du gisement. Nous y avons trouvé quelques relicta interessants; par exemple, un très bon burin grattoir, un fragment de bois de renne à terminaison ovale, probablement extrémité d'un lissoir, - un petit bloc de "alcaire ayant trois petites cupules. -.. une autre pierre a cupule, - une molette en grès (de la région de Brive probablement), de forme elliptique. ayant servi visiblement à broyer et à lisser (les deux facettes extrêmes sont un peu écrasées, mâchées, et les deux faces latérales sont lissées), une moitié de godet ayant fait palette à ocre. Ces objets nous paraissent avoir appartenu à la couche magdalénienne, par comparaison avec l'Abri Reverdit. au Vallon des Roches de Sergeac ${ }^{1}$. Notons aussi que les déblais ont donné surtoui des dents de renne, tandis que la couche aurignacienne-a fourni surtout du cheval.

L'horizon anurgnacien comportait trois fovers, qui n'étaient pas à la même hauteur à cause de la présence de plusieurs blocs, devant provenir du fronton de l'abri, tombés v'raisemblablement vers la fin de l'épo-

(1) F. Delace, Les roches de Sergeac, dans L'Anthropologie, 1935, nos 3-4, p. 281$317,16 \mathrm{fg}$. 
que moustérienne. Ces pierres élaient enrobées dans la couche archéologique. La couleur générale de la couche était rougeâlre '.

a) Outillage osseux (os et bois de renne) (fig. 6). - L'objet caractéristique est la pointe d̀ base fendue, représentée par cinq fragments d'aspect très net, dont le principal indicjue le type losangique. On discerne bien le travail du silex qui a lendu la base, et l'amincissement par râclage des deux faces. Il y a là confirmalion de ce qui a été observé ailleurs pour la fabrication de ce genre d'objets.

Des fragments de bois de renne montrent aussi la fabrication de futs de sngaies: sciage en desius el en dessous, enlèvement de matière pour préparer la lorme subcylindrique, et sciage en travers pour détacher l'objet. Une base de sagaie ovalaire a une face lisse, et l'autre restée rugueuse. Un fragment de sagaie montre une pointe nettement, cylindrique.

Plusicur's poinçons, soit entiers, soit fragmentés, dont certains faits d'une esquille d'os finement travaillée. Notons un poinçon arqué, un poinçon à double pointe.

$\mathrm{Au}$ total, l'outillage osseux ćlail peu abondant, et en général fragmenté (chûtes de blocs sur les foyers). Mais il est assez net pour indiquer l'Aurignacien typique (type d'Aurignac).

b) Outillage lithique. - Un assez grand nombre de pièces sont tirées de silex colorés d'un très joli aspect: bleu noir, jaune translucide, jaspe verdâtre tacheté, rouge violacé, blanc moucheté, miel clair, cire foncée, jaspe rouge, calrédoine fumée, calcédoine rosée, jaspe-agalhe à rubans ou à zones, etc... On a noté dans d'autres gisements la prédilection des Aurignaciens pour ces couleurs qui flattent l'oil. Nous avons signalé ce goût des teintes variéses ot même bigarrées dans nos trouvailles du platean de Belcayre-Haut ".

Les lames sont particulièrement intéressantes; le débilage des lames est un des caractères de ce niveau. Ia longueur dípasse asse\% souvent $10 \mathrm{~cm}$; elle va jusquà 15 et $16 \mathrm{~cm}$. Les plus larges ont 40 et 15 millin. L'ípaisseur est fréquemment faible; la plupart sont planes, flusieurs arqués. Dans des ras nom-hreux la retouche est excellente, affectant lout le pourlour, arre une intensite variable, parfois tres courte et légire, parfois longue sorte de souvenir moustérien), ici très plate, là plus épaisse. Jexlémité nposié an talon est généralement ovalaire. Les lames ont fourni de bons couteaur el de délicals canifs. Il est d'autres couteaux faits sur un éclat peu épais, dont le dos a cité retouché et même rabattu. Peu de lames paraissent avoir été taillées en vue de constituer des pointes de sagaies on javelots; ce genre d'engrins citait élabli de préférence avec du bois de renne. Cependant, le haut du niveau nous a donné une pointe le La Gravelte, plutôt massive, avec dos fortement abaltu, extrémité acérée.

Les lamelles à dos rabattu étaient trò̀s yen nombreuses; le còté coupant présente des traces d'usage.

Belcayre doit aussi au débitage de la lame de trìs bons grattoirs, dont la longueur va jusqu'à $0 \mathrm{~m} .16$, avec une épaisseur pourtant faible, le pourtour retouché, le talon accommodé (fig. 7 ). Les formes larges sont plutiot rares. Quelques gratloirs ont une coche, soit sur un eoté de la tête, soit sur un des bords longs. Plusieurs ont un bord retouché, et l'autre esquillé comme le serait un couteau usagé. Les détails morphologiques sont si nombreux fu'il est impossible de les

(1) Un de ces blocs avait $2 \mathrm{~m}$. de long, $0 \mathrm{~m}$. 60 de largeur, $0 \mathrm{~m}$. 40 d'épaisseur; un autre, tout noirci par la fumée et la suie, mesurait $2 \mathrm{~m} .70,0 \mathrm{~m}$. 80 et. $0 \mathrm{~m}$. 30 . Les foyers étaient gras, pleins d'os brulés et de morceaux de silex; un de ces foyers, soutenu par un des grands blocs, donnait l'impression qu'on y avait carbonisé la majeure partie d'un animal (cheval?). Line partie de la base de la conche r'eposait sur une sortr de dallage de pierres assez plates.

(2) V. notre note dans le volume de l'(Onzirme Session du Congris l'réthistorique ('érigueu.c), 1994, p. 386. 


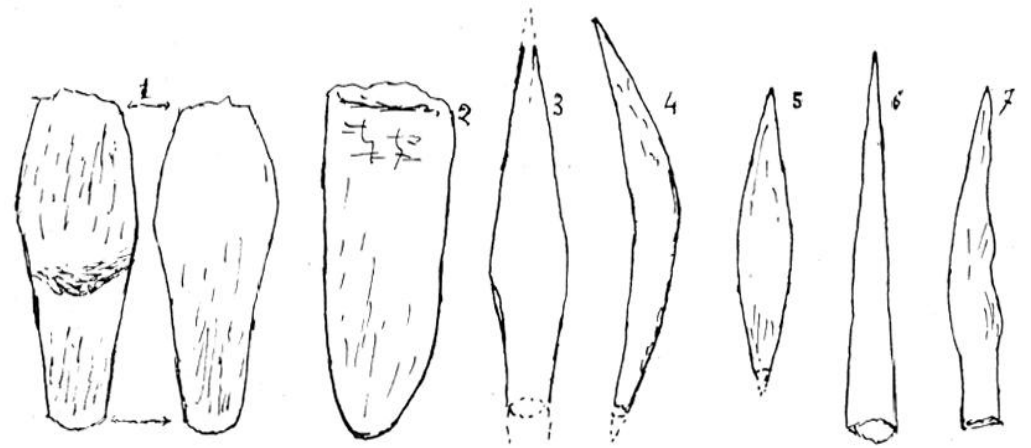

Fra. 6. - Abri de la Métairie. durignacien. 1: Fragments de "base fendure" 2: Biseau de sagaic. 3: Pointe de trait. 4, 5, 6, 7 : Poincons. (Outillage usselux.)
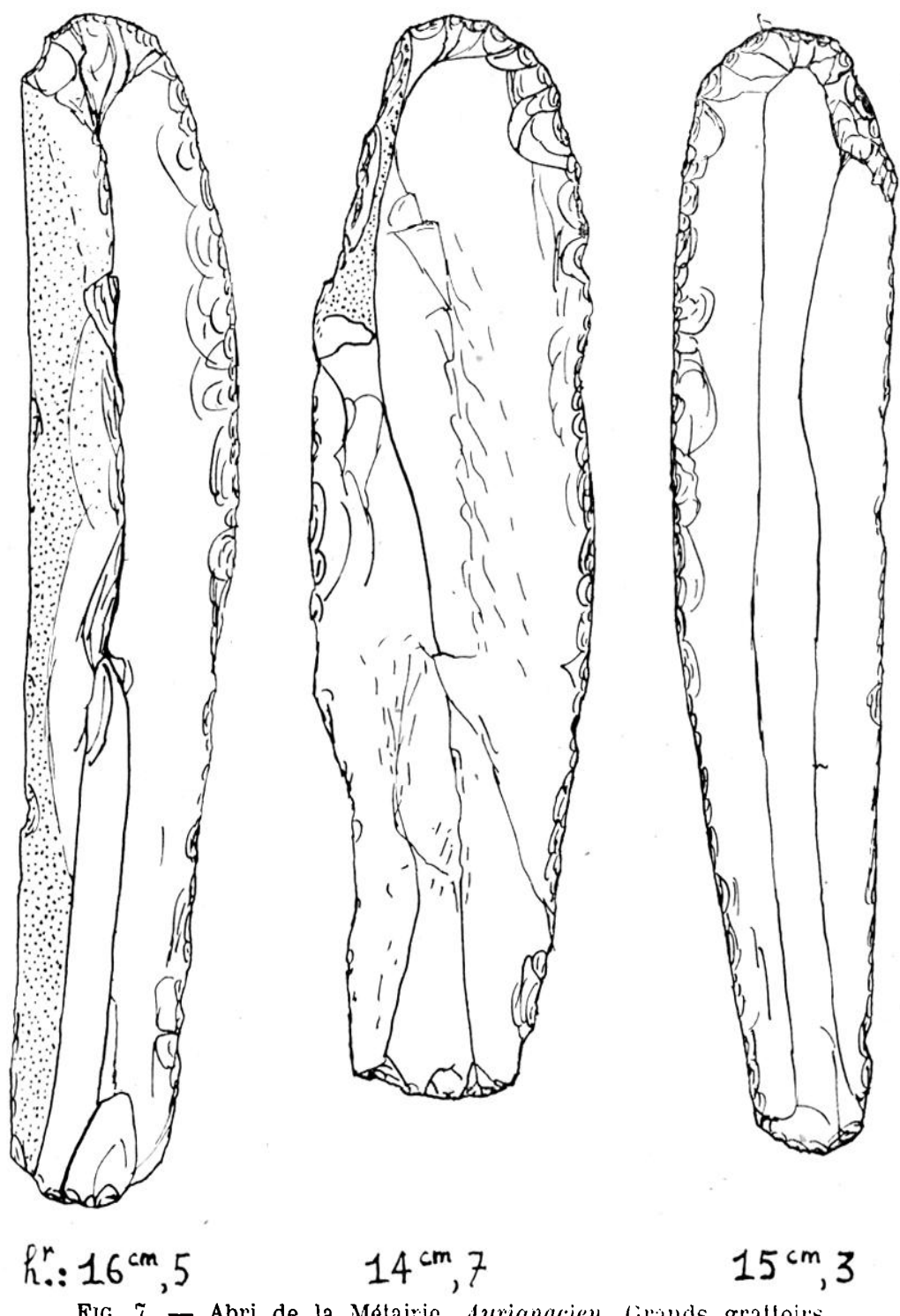

Fus. T. - Ahri de la Métairie. Aurignacien. lirands grattoirs. 
signater ici. Notons cependant que des lames montrent un grattoir concave, d'autres rectilignes et quelques-uns en "éventail". Les grattoirs doubles n'étaient pas nombreux; ils sont retouchés ou esquillés sur tout le pourtour. Les formes approchant du type circulaire étaient aussi en petit nombre.

A propos de l'outillage lamellaire (fig. 8), il faut noter des lames tronquées, en forme de parallélogramme rectangle, parmi lesquelles on remarque une accommodation voulue sur la troncature, et retouche ou utilisation sur un ou sur deux bords.

Les grattoirs à museau, plus souvent sur éclat que sur lame, ont été nombreux; les formes en sont très variées; dans bon nombre le museau est très bien dégagé; quelquefois il s'affinc pour devenir une sorte de bec. Assez souvent il forme la terminaison d'une pièce en carène. Les grattoirs carémés sont aussi un aspect remarquable du gisement, très variés de largeur, de hauteur, de confection; il y a plusieurs spécimens très bons, dont la forme en dòme ou en pyramide est fort réussie.

Dans les burins, les variétés sont nombreuses: becs de flûte, burins à pans multiples, burins à pans retouchís, elc... Les burins sur angle de lames sont nombreux et intéressants; nous en notons un. à cause de sa dimension, qui a $10 \mathrm{~cm}$. 5 de long sur $4 \mathrm{~cm}$. de large. Les petits "burins de Noailles" étaient peu nombreux. Les burins busqués sont en quantité assez grande pour constituer un trail important de ce niveau. Quelques perroirs de qualilé normale, sur bouts de lames, ou sur éclats: quelques-uns sont assecz forts pour être considérés comme des tarauds (fig. 9 et 10 ).

Des éclats triangulaires ont des usages variés (pointes, couteaux, etc...), certains sont retouchés de telle sorte qu'on doit y voir mieux que des outils de fortune. Les coupoirs, les scies, les tranchets n'ont rien de spécial. Racloirs nombreux, de toutes dimensions; bord soit rectiligne, soit convexe; coches (dont certaines lortes), assez fréquentes sur des còtés de racloirs, dos naturels ou retouchés; deux formes amygdaloïdes (pseudo coup-de-poing); quelques rasoirs en quartz.

Plusieurs pics (longs de 8 à $9 \mathrm{~cm}$.), en général globuleux, avec pointe préparée, ou, tout au moins, écaillée par l'usage; ce sont des outils de sculpteur's ou de faiseurs d'anneaux et de cupules. (Nous en avons trouvé dans les rejets des fouilleurs précédents.

Décompte des pièces principales de l'industrie lithique:

Grattoirs 90 (dont 31 sur lames, 17 sur éclats, 5 rondâtres, 9 doubles, 16 carénés, 12 à museau); - burins 75 (dont 39 types ordinaire, 10 busqués, 26 sur angles); - burins-grattois $4 ;$ - racloirs $36 ;$ - rabots $13 ;$ - lames retouchées 27 ; - lamelles rabattues 27; - couteaux 27; - scies 17; - perçoirs 5; - pics 4; - pièces triangulaires 12; - pièces quadrangulaires 9.

c) Parure. - Un peu de manganèse et davantage d'ocre représentent les matières colorantes; pas de motifs décoratifs découverts; - un coquillage percé d'un trou peu régulier; deux dents percées (une canine de renard; une incisive de cheval).

\section{C) Niveau magdalénien ancien.}

A la partie supérieure du gisement, des foyers magdaléniens buttaient contre la falaise; l'étendue en avant était très limitée, la pente du talus ne laissant qu'un faible espace horizontal; de plus, la partie antérieure avait été enlevée par des chercheurs divers. Le côté amont, seul respecté, a fourni un bon nombre de pièces intéressantes, malgré son faible espace. La couche, épaisse de 10 à $25 \mathrm{~cm}$., était écrasée en plusieurs points par des blocs, notamment le grand bloc à anueaux dont 

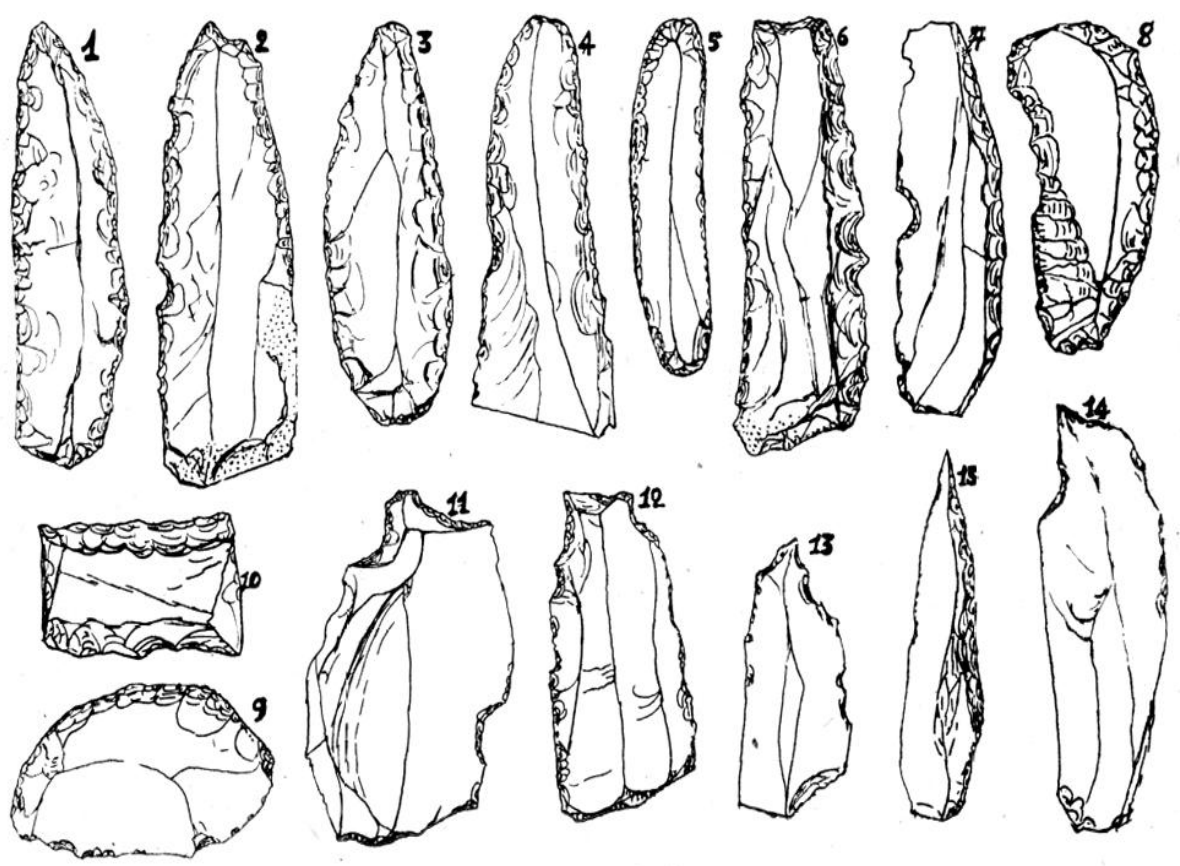

Fig. 8. - Abri de la Mêtairie. Aurignacien. 1, 2, 3, 4, j: Lames relouchées.

$6,7,8,9$ : Couteaux et scies. 10: Lame tronquée retouchée. 11: Grattoir à museau. 12, 13, 14: Burins divers. 15: Lance acérée, tỵe Gravette.
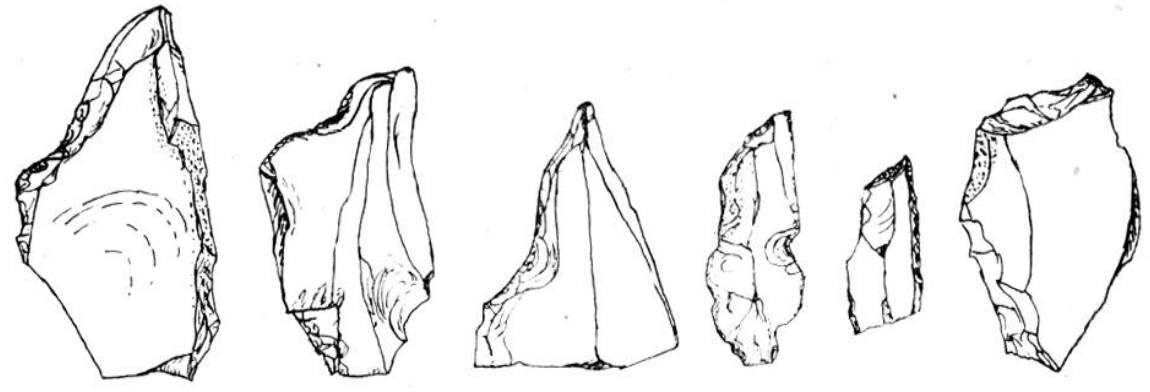

Fig. 9. - Abri de la Métairie. Aurignacien. Divers types de burin.
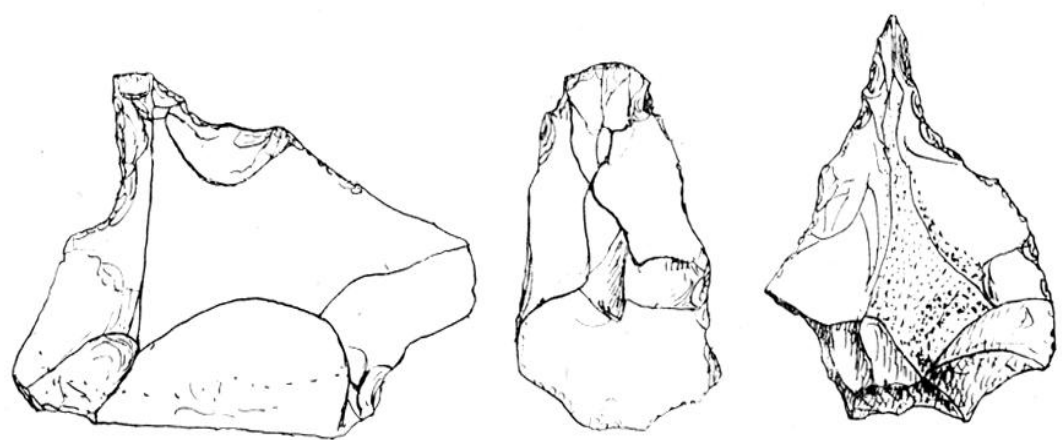

Fig. 10. - Abri de la Métairie. Aurignacien. Outils pour piquer el tarauder la pierre. 
nous parlerons plus loin. Les objels en bois de renne étaient presque lous brisís.

La famme est neltement domince par le renne (dents, sabots, bois abondants); elle comporte de petits rongeurs, des oiseaux, des poissons, sans particularité remarquable. Nous avous indiqué la prédominance du cheval daus l'horizon sous-jacent.

Dans l'outillage en silex, absence de pointes de la Gravette, de pointes a cran, de feuilles de laurier. Dans l'outillage en os et bois de renne, pas de bases fendues, pas de harpons. Le niveau étudić ici apparâit done comme appartenant an Magdalinien ancien, avec l'impression d'une certaine contimuilí aver l'Lurignacion en ce qui connerie le silex. La présence de pierres à ammanx ol de pierres a rupules mppolle à la fois l'Aurignarien de Sergeac (abris Blanchard el bidon) el de Fongal, et le Magdalónien ancien de l'abri Reverdit (Vallon des Roches de Sergeac). Pour la connaissance du Magdalénien à ses clébuls, nous croỵons ulile de signaler des particularités avec plus de détails que pour le niveau aurignacien. mais plutôt pour l'outillage osseux que pour l'outillage lithique:

i) Outillage lilhique. - Eclats de quntz utilisés: - petits nucléi pyramidaux ou en dos d’àne, pouvant servir de rabots cun petit rabot double, long. 0,045. haul. 0.035); - ́rlals atypiques vlilisís de fagons diverses (dont un éclat long de 0,125, avec talon bien aceommorlé, forles traces d'usage a l'ex(rémité oblique).

lames. minces, plates, plus ou moins ulilisées. Lames relouchées. dont une a un rétricissement tendant au pédoncule.

Lame's appoinlées (dont une retouchée légirement à droite, plus fortement à gallohe).

Burins de tyjes divers, dont plusieurs avec relowehe.

Percoirs of poinrons, do types cortects. sans réussite particulière; à noter un fort perẹoir bien dégagó par deux coches sumétriques.

Gralloirs: sur lames asser rares (un exemplaire de lame extrêmement mince); gratloirs sur éclats, dont quelques-uns concaves; un colat rondâtre (dimensions $0.04 \times 0.03: 2)$. bombé rappelle le carenci: gratloirs a bec ou museau, de petites dimensions (longs de 0,05 à $0,0 \tau$ ), parfois à profil caréné. - Rabots de labrication peu soignce.

Microlithes: nombreuses limolles à dos rabaltu, visiblement utilisées, ayant parfois un bout faronné en poinle: un fragment esl une survivance de La Gravette (dos fortement retouché, pointe aiguë vigoureuse); petits burins (longs de $0,019,0,024$, etc.).

Deromple des pieces principales de lindustrie lithigue:

Gratloirs 31 (dont 9 sur lanes, 15 sur éclats, 1 rond, 1 double, 5 carénés, 3 it museatu): - burins 31 (21 ordinaires, 6 sur angles 4 a pan retouche); - buringrattoir 1; - racloirs 13; - rabols 10: - muclei taillés e'n pyramide 3: lames refouchérs 5: - lamelles raballues 11; - couleaux 16; - scies 6; perçoirs 4; - quarlz ulilisés 4 .

b) (Figure 11): outillage en bois de renne ou os. - Le travail du sciage el du débitage de ces matières est précisé par de très nombreux fragments. Ainsi, pour la fabrication des baguettes, on voit conment on sciait un bois par deux traits longitudinaux poussés à la profondeur de jo a $8 \mathrm{~mm}$., après quoi on détachait la baguette qui avait 3 faces planes et une face ruguense quon régulari- 
sait. Nilleurs se voient des rainures doù l'on a extrait la matière des aiguilles ou des poinçons:

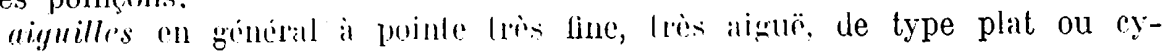
lindrique (trouvées par fragments);

petits poincoms, soit en os (dont un nettement lissé et poli), soit en bois de reme (dont, certains montrent les traces du débitage par sciage et des facette; (fu'trait la baguetfe avant d'ètre rendue cylindrique);

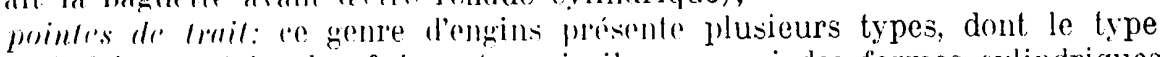
semi-cylindrique est le plus fréquent; mais il $y$ a aussi des formes cylindriques w légèrement subcylindriques. Les pointes ne sont pas trè fines; whe pièce longue de $13 \mathrm{~cm}$. est appointée non aiguë; une pièce, légèrement arquée (long. $11 \mathrm{~cm})$, montre a sa pointe les coups de taillage en biais et en travers; une forte piece (long. 0,13$)$ a un bout non aigu, inacheve en quelque sorte. Dans quelques autres as, le bout est finement aninci et appointe: telle, une longue sagaie (long. 0,165), remarquable par sa faible épaisseur (maxima 0,011). Parmi les bases: une base conique (sagaie de 0,165$)$; un biseau long de 0,05 sur la pièce longue de 0,11 . - un biseau double, a paris lisses, longs de $0,0.42$ pour une pièce longue de 0.13 ;

poigmard en os, long de 1,125, avor puignéc faite dune épiphyse accomodée, fiil copais, pointe massive:

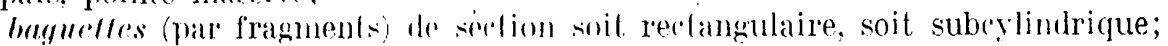
lissorirs al sulull's, dont un l'agment à extrémité ovale, avec une face légèrement bombéc, l'autre légèrement concave; - une pièce en os (trìs abîmée) de forme plate et mince (coulcau a papier) avec un bout ovale et les cotés arrondis;

coins ot ciscaux, dont un fragnent de gros outil à fût cylindrique (diamè-. tre moyen 0,01\%), dont l'extrimité, à dessous plat et dessus bombé, finit en ovale mousse.

Ornementation: série de 15 potits larils nhlipturs. parallèles, sur le dos d'un roinçon; 6 petites encoches sur la partie inlérieure d'une sagaie demi-ronde; sur la face la plus large du poignard, longs trait's parallèles, recouprs par de petits traits obliques; - sur la face plate de la sagaie arquee, une rainure longue de $0 \mathrm{~m}$, 025, et, sur le dos, une série de chevrons inégaux, racrordés par uin trait tracé selon l'axe de la pièce. Cette ornementation, simple et uniquement linéaire, répond bien à co que l'on sait du Magdalénien ancien.

c) Itarure. Ocres el manganise frequents; galet ayant servi de palette pour ocre rouge; molettes en grès gris, de forme elliptique, dont les faces planes sont usagées; - un petit galet plat, perfor'i pour suspension, en bon ciat, et un autre petit galet dont le trou est cassí.

d) Pierres à anneaux et à cupules - A l'extrémité de la couche grisait un tris gros bloc (dimensions principales: $1 \mathrm{~m}$. 70 et $1 \mathrm{~m}$. 40 ) qui roravilil lat couche. Dégagé et retourné, re blor a montré des particularilés intriressintes, que nous décrivons en allant de gauche à droile:

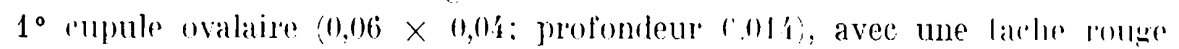
à côté; - - ?" grand anncau sur l'arête du bloc, anse liarge de 0.035 , cipaisso de 0,$045 ;-3^{\circ}$ en contrebas, grande cupule ovale $(0,18 \times 0,12)$, sans mofondeur. $(6 \mathrm{~mm}$.$) : - 4^{\circ}$ petit anneau brisé; - - " auprès de l'arête, ébauche d'anneau inachevi; - $6^{\circ}$ chauche damneau moins avancée; $-7^{\circ}$ ébauche d'ammean, plus poussée; - - 8" chauche encore vacue; - $9^{\circ}$ petit anneau brisé, sur la face large du hloc. Outre ce graml bloc ainsi e'rible de traces du travail de l'homme, nous avons trouvé dans les déblais une pelite pierre creuscée d'une cupule bien formée, el une autre pierre, de faible volumr. oi se voient trois petites cujules.

Rappelons l'existence damneaux of de cupules dans le vieux Magdalcinien de l'abri Reverdit (Roches de Sergeac) que nous avons publiées et figuries jar des photos (voir plus haut, p. 9. n. 1.;. 


\section{III. - L'ABRI DU RENNE'1}

A 150 mètres à l'ouest de la métairie de Belcayre, une zone de rochers presque entièrement éboulés celait un gisement abondant, peut-être remué en surface çà et là par des ramasseurs de silex. Ayant découvert ce gisement en 1924, nous y avions fait un sondage développé, qui donnait des résultats très encourageants, quand l'indiscrétion de certains chercheurs vint bouleverser nos travaux et rendit presque inutile au point de vue scientifique la continuation des fouilles. Cependant, notre première récolte était assez abondante pour permettre une interprétation certaine. Le gisement appartient à l'Aurignacien, et, semble-t-il, à la face moyenne de cet horizon "

Immédiatement sous la terre végétale, se présentent une grande quantité d'éclats, de nucléi, de silex taillés, d'os brisés, de galels, etc... Puis vient un éboulis de pierrailles n'ayant qu'une quinzaine de centimètres d'épaisseur, enrobant une grande dalle ornée d'une figure gravée. Sous ces pierrailles, une deuxième couche est aussi riche en silex de mêmes types.

Nous avons décrit précédemment $\$$ la gravure, qui représente un animal de la famille des cervidés, très probablement un renne (la tête étant abîmée), ou plutôt une femelle de l'espèce renne (fig. 5). Le contour du corps a été établi au moven d'un pic de fort calibre, creusant des rainures irrégulières ${ }^{4}$. Le style est évidemment archaïque. schématique avec le maximum de détails. Dans cette silhouette en profil absolu, et d'une immobilité rigide, deux jambes seulement sont figurées; rependant un air de vérité générale est assez frappant.

On doit comparer cette gravure à celles de Fongal ( $\mathrm{C}^{\text {ne }}$ de Peyzac), de La Grèze ( $\mathrm{C}^{\text {ne }}$ de Tursac). du Terme-Pialat (St-Avit-Sénieur), et à d'autres que nous avons indiquées. On notera aussi un air de famille avec des gravures de La Ferrassie également naïves. Ia figuration en profil absolu, très simplifié, n'est pas absolument spéciale à l'Aurignacien; mais, hors de cet horizon. on ne peut la trouver que dans la phase première du Solutréen ou du Magdalénien. Le milieu d'où vient le bloc en question ici est strictement aurignacien.

(1) Appellation tirée de la dalle gravée que nous avons trouvée dans ce gisement.

(2) Lors de l'incident facheux auquel nous faisons allusion, nous nous sommes bornés à une "prise de date", insérée dans le Bull. de la Soc. Préhist. Française, année $1924, \mathrm{n}^{\circ} 10$, p. 240.

(3) Congrès Préhist. de France, $X I^{\circ}$ session (Périgueux), 1934, p. 388-392.

(4) La tête de l'animal ayant êté très abîmée par un accident contemporain fut restaurée au moyen d'un burin traçant un sillon !éger, très différent du premier procédé. - Longueur de l'animal: $0 \mathrm{~m} .42$; haut. maxima: $0 \mathrm{~m}$. 37. Dimension de la pierre: $1 \mathrm{~m} . \times 0 \mathrm{~m} .50$. - Cette pièce, conservée au Musée National des Ẹ̌zies, a été reproduite dans Les Artistes Préhistoriques de MORIN-Jean (1933), p. 57. 


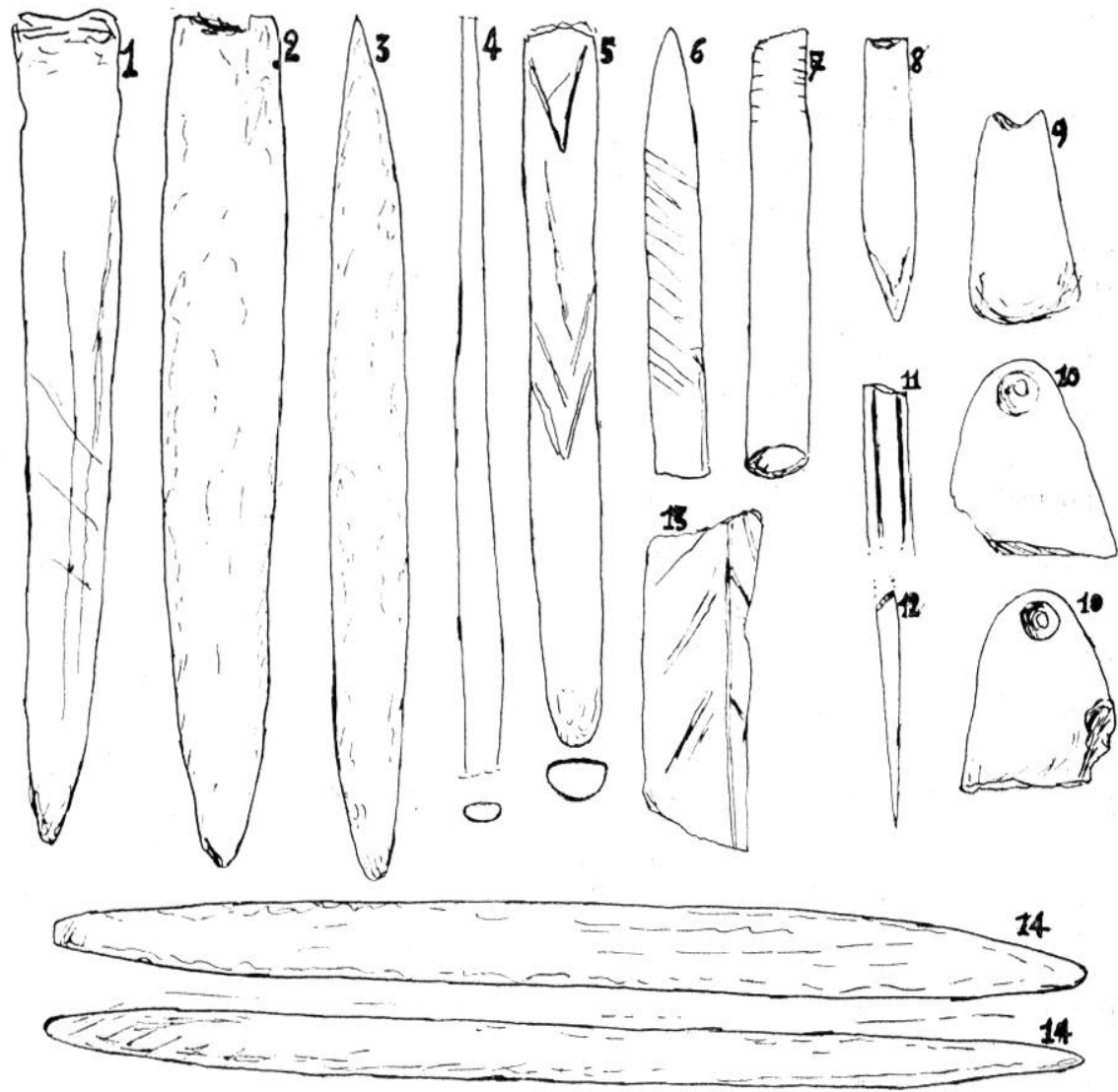

Fig. 11. - Abri de la Métairie. Magdalénien.

1: Poignard ou fort poinçon. 2: Poignard. 3: Sagaie. 4: Alène. 5: Sagaie subcylindrique avec chevrons. 6: Fragment de sagaie avec traits incisés. 7 : Fragment de baguette ronde avec coches. 8: Base conique: 9: Pendeloque cassée. 10: Pendeloque (les \& faces). 11: Baguette à cannelures. 12: Fragment de poinçon. 13: Baguette plate avec camnelures et traits incisés. 14: Longue sagaie (de face et de profil).

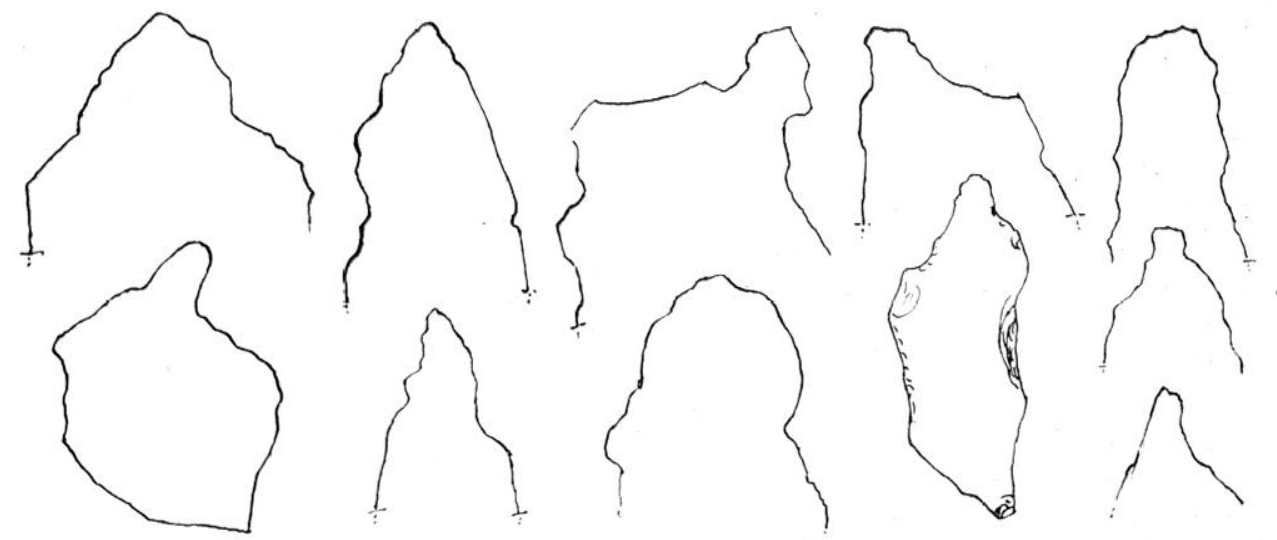

Fig. 12. - Abri du Renne. Choix de bases de grattoirs à museau. 
Notre première tranchíe ne nous a pas procuré d'oulillagge osseux, sauf un très court f'ragment de baguelle ronde. Par contre, l'outilluge liihirgere dait abondant el varié:

Lames nombreuses, mais peu de remarquables. Une seule fris lomme, a bords bien retouchés, de forme lancéolée (long.: $0 \mathrm{~m}$. 12). Pas do relouche vaiment abrupte; généralement, un bord est relouche plus fortement que l'aulre qui est souvent plutòt utilisé que taillé. Plusieurs hases de lames onl idr rétrócies de façon à former un pédoncule, ou, tout au moins, un talon accommorlé. I'extrónité opposée a la base est parfois retouchice. Quclques coches enlament un bord de l'objet. Une lame finement appointée a aussi servi comme rouldaull.

bes eclats lamellaires ont fourni des routeaux ou des rompoirs. ayanl te dos naturel ou arrangé pour la place des doigts manouvranl loulil. Cirtaines de ces lames ou éclats coupants ont une extrémité retaillóc on josudu-grattoir; cette extrémité nous paraît jouer le ròle de poignée.

Grattoirs sur lames a section triangulaire ou traproüdale: sins retouche

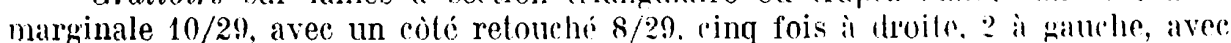
deux côtés retouchés 10/29. Grattoirs sur colats lnogs (25): sur ćclals rourts, aussi larges que longs, et même plus lareres qur longs, parfois aver un amincissement faisant pédoncule (9)'t pirensi. Giralloirs gonds (7). plus nu moins ro fouchés sur le pourtour.

Plusieurs eouteaux ef gralloirs ont dos coches: mais il y a do virilables outils faisant yralloir conrare (15) pieres), dont un long cerlal (1) 111.125$)$. presen-

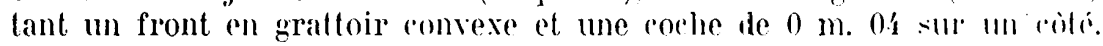

Nombreux carénés ef rabols. aree diverses formes de lansilion. à retonche longue; pièces en éventail (un double civentail); nuclói do lon'me haule (denti un jyramidal), transformés en gros carénés ou en rabots: werlle "subot de cheval"; des micro-rabots, doubles ou simples. Ies carénés donl la parlie ouvrante est en ogive sont nettement plus longs que larges.

Tres forte proportion de muse'aur (fig.l2) (petits blocs: polits nuclei; colats longs ou rondâtres): 10 museaux arrondis. fo museaux allongris of mrosque effilés selon l'axe de la pioce: 5 museaux déjotós vers la droite, i déjelés sur la gauche; 4 approchant de la forme d'un taraud. Ces pièces ont gimiralement les côtés retouchés; la plupart sont hombées, mais quelques-umes plate's. L'abondance de. ce genre d'outils constitue un álément tris important de ere gisimment.

Burins: burins droits en bec de flôle. (26); burins sur angles de lames

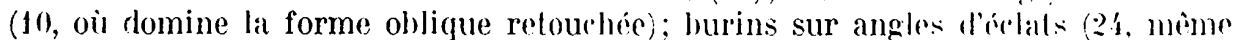
observation; notons un éclat épais temine par un fort burin dígage all moyen de deux encoches très mar(fuces); burins husqués (8. le coito husqui est loujours a gauche, et le pan droit est vertical ou légirement obliguej. ()n retiendra la présence de re genre de burins commo caraclìre de ce gisemont. - Burins-qratloirs: 3 , dont deux de bomne qualité.

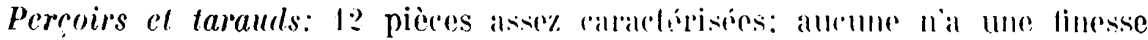
de facon remarquable.

Echals triangulaires. rapjedant reux qui fournissent ha "primlo" mousté-

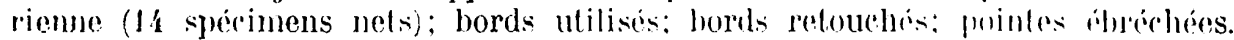
votons une piece avant tout le bord gauclue retouché en des:us. la demi-longueur du bord droit retouchi en dessous, de sorte que cas rolomelos inverses se rejoignent au sommet de l'outil.

Scic's (2'), visiblement utilisées: tranchant rectiligne ou arquí, prósonlant parlois des dents résultant de l'usage; plusicurs sont des inlals rectangulaires à dos épais.

Racloirs (30), presque toujours plus longs que larges; quelours-uns rappellent la retouche moustérienne; le còté auvrant est soit convexe, soit rectiligne 
(un cas a bord sinueux): un racloir double: des formes complexes (par ex.: terminaison par un nez accoste dune coche; -- talon arrangé en forme de grattoir) ${ }^{1}$.

Décomple des pièces principales de l'oulillage lithicue:

Ciralloirs 184 (dont 29 sur lames, 25 éclats longs, 29 éclats courts, 7 rondâtres, 15 concares, 13 carcinćs. 66 a museau); - burins 58 (dont 26 ordinaires, 24 d'angle, 8 busqués; - lurins-grattoirs 3; -.. racloirs 27; - rabots 8; lames retouchées 29; -- coulırur 18; - scies :6; - perroirs 12; - pieces triangulaires 14; - pics 2.

Les gisements de Belearre-Bas, depuis le Gîle inondé placé sous le chàleau de Belcarre, jusqu'à 150 mètres au delà de La Métairie. sonl tous paleolithiques. Ils apportont des renseignements sur l'outillage d'un Moustérien évolué, de l'Aurignacien moyen (phases I et III, semble-t-il) et du Magdalénien ancien (phase II ou III). En particulier le dernier gisement, s'il arail pu ètre fonillé correctement. aurait apporté une documentation importanle pour la morphologie du matíriel lithique. On retiendra parmi les éléments intéressants la dalle gravée, bon exemplaire de l'art aurignacien. Ce's gisements n'ạant donné aucun niveau solutréen. on retiendra aussi la superposition du Magdalénien ancien à l'Aurignacien avec une asse\% faible sćparation et la présence des anmeaux of des cupules.

Franck Masitik.

[1) labondante bibliographice des travaux de M. Franck Delage, nous extrayons les titres suivants qui concernent particulièrement nos Antiquités Nationales:

LAMTIROPOLOGIE:

L'abri de La Gauberl (Dordognc), XXXII, 1933, p. 445-451. fig.

Les roches de Sergeac (Dordogne), XI. 1935, p. 281-317, ill.

L'âge du bronzé en Limousin. I.III, 1949, j). 563-567.

BULLETIN ARCHEOLOGIOUE DU COMITE IOES TRATALS MISTORIQUES:

Marques de potiers en Limousin. 1930-1931. p. 333-335.

Sépulture gallo-romaine ì La Gorec (Haute-Vicnne), 1931-1935, p. 489-492. Autel gallo-romain ì loutiors (Haute-Viemm), 1934-1935, p. 509-513.

Sépultures yallo-romaines de la Correze. 1938-1940, p. 499-505.

Deux monuments antiques e'n Ilaute-lienne. 1938-1940, p. 511-513, 3 pl., ill.

Fouilles aur ruines gallo-romaimes du "Clhaleau des Cars" (en collab. avec I. Prieurj, 1938-19.90, p. 515-5:01).

Inventaire des marques de poliers gullo-romains trourés à Limoges et dans li. Haute-Vienne, 1941-1942, p. 471-497.

Jarques de potiers gallo-romains recueillis dans la Corrize, 19:11-1942, p. 499-504.

(1) Comm. pour la partie aurignacienne du gisement dit de La Métairie, nous pouvons indiquer l'abondance des couleurs variées: divery jaspes, silex gris à zones concentriques blanches, jaunes ef sienne-clair, mastic i bandes ou raies jaunatres, lilas, Inauves; gris clair à écharpe blanche; blanc ivoire, cire, elc... 


\section{BULLETIN DE LA SOCIETE ARCHEOLOGIQUE ET HISTORIQUE DU LIMOUSIN:}

Dolmens et druides: Le dolmen de Breuil (IIaute-Vienne), LVIII, 1908, p. 17-43. Souterrains de Limoges. LVIII, 1908-1909, p. 640-645.

Archéologie préhistorique: Dolmen de la Villedicu, hach's néolithiques, LIX, 1909, p. 5-13, ill.

Mélanges d'archéologie limousine: Dolmen de Reneix; urne de Châteauneuf; découvertes gallo-romaines à Limoges, LIX, 1910, p. 337-40.1, ill.

Mélanges d'archéologie limousine: Nouvelle série: Inventaire de's dolmens de la Haute-Vienne; tumulus de Saint-Germain-les-Belles; haches en bronze; sépultures de La Croisille, LXII, 1913, p. 237-252, ill.

Le souterrain de Mortorat (Creuse), LXV, 1916, p. 5-12, plan.

Excursion archéologique en 1924: Dolmen du Puychaud; ménhir de Centurat; camp de Saint-Gence; cippe de Javerdat, LXXI, 1924, p. 606-612, ill.

Le camp de Villejoubert, LXXII, 1927, p. 123-152, ill., pl.

Excursion archéologique de l'année 1927: Epoqucs préhistorique 'l gallo-romaine, LXXII, 1929, p. 446-494, ill.

Excursion archéologique de 1928: monuments préhistoriques ct époque galloromaine, LXXIII, 1930, p. 312-359, ill.

Le vase en terre sigilléc de La croisille, LXXIII, 1931, p. 495-500, ill.

Les monuments mégalithiques de la commune de Saint-Auvent, LXXIII, 1931, p. 599-611, ill.

Les fouilles de la villa dintone, IXXIV, 1932, p. 1-22, ill.

Excursion archéologique de 1930: époque préhistorique ct époque gallo-romaine; mines antiques, LXXIV, 1932, p. 191-206, ill.

Le Jupiter de Jioux, LXXIV, 1932, p. 219-235, ill.

Les ruines gallo-romaines de Souffas, commune de Vicy (Haute-Vicnne), LXXIV, 1932, p. 261-264, ill.

Notes archéologiques: L'Artige; Planchemonton; souterrains de Fromental (HauteVienne), LXXIV, 1933, p. 271-286, ill.

La sépulture gallo-romaine de Planchemouton. LXXIV, 1933, ]. 282-286, ill.

Excursion archéologique en 1931: époques préhistorique et gallo-romaine. Le préhistorique et le gallo-romain à Be'ssines, Chateauponsac, Folles, Fromental et Razès (Haute-Vienne), I.XXIV, 1933, p. 478-520, gr.

Excursion archéologique de l'annéc 1932, LXXV, 1934, p. 208-247, ill.

Fouilles de puits gallo-romains ì Chassenon (Charente), LXXVI, 1936, 1. 599622 , ill.

Antiquités gallo-romaines à Roussac et à Balledent, LXXIX, 1942, p. 327-340, ill.

Notes gallo-romaines (Haute-Vienne): Inscriptions et vases céramiques (en collab. avec J. Tyl): Vase sigillé découvert à Limoges, LXXIX, 1942, p. 349-352, ill.

Inscription gallo-romaine découverte à Limoges, LXXIX, 1942, p. 352-356, ill. Inscription gallo-romaine retrouvée, LXXIX, 1942, p. 356-361, ill.

Inscription gallo-romainc du Buis, LXXIX, 1942, p. 362-364.

Vestiges gallo-romains à l'Usine à gaz de Limoges, LXXX, 1944, p. 240-250, ill. Notes d'archéologie gallo-romaine: $16^{\bullet}$ série, LXXXI, 1946, p. 213-226, ill.

Notes d'archéologie gallo-romaine: $17^{\circ}$ série: Fouilles à Chassenon, LXXXII, 1947, p. 151-174, ill.

Les dolmens de la Haute-Viennc, LXXXII, 1948, p. 107-413, carte.

Les cultes gallo-romains en Haute-Vienne, LXXXIII, 1949, p. 121-129.

Le dieu accroupi d'Aigueperse, LXXXIII, 1949, p. 35-38, gr. 
BULLETIN DE LA SOCIETE HISTORIQIE FT ARCHEOLOGIQTE DU PERIGORD:

Le souterrain-refuge do Loubatour. LIX, 1932, p. 142-145, pl.

L'abri de la Souquette ì Serge'ac (I)ordogme), IX, 1938, 1. 10.1-1:6, ill.

Rochers de l'Acier ì Sergenc. IXXY, 1947, p. 92-97, ill.

BULLETIN DE LA SOCIETE PREHISTORIQUE FRANCAISE

Les dolmens de la Haute-Vienne, XVIII, 1922, p. 84-88.

L'oppidum de Villejoubcrt (en coll. avec C. Gorceix), XX, 192'4, p. 208-229, ill.

Fosses ovoides en Limousin, XXVIII, 1931, p. 340-346, ill.

Un tumulus à fosses multiples ì Forgès (Corrèze), XXIX, 1932, p. 250-253, ill.

Le camp de Cornouin, XXXII, 1935, 1. 386-387, ill.

Bronzes celtiques trouvés en Limousin, XXXIII, 1936, p. 525-528, ill.

Bracelets hallstattiens de: la Corrèze, XLVI, 1949, p. 431-432.

BULLETIN DE LA SOCIETE SCIEYTIFIQUE, HISTORIOUE ET ARCHEOLOGIOUE DE LA CORREZE:

Le Gallo-romain en Bas-Limousin. I.X, 1938, p. 113-142.

CONGRES DE LA FEDERATION DES SOCIETES SAVANTES DE CENTRE:

Les sépultures gallo-romaines en Haute-Vienne. 1938, p. 242-25\%.

CONGRES PREHISTORIQUE DE FRANCE:

Dolmen inédit de la Villédieu, Beauvais, 1909, p. 386-392, ill.

Inventaire des grolte's al abris préhistorique's de france, Angoulême, 1912, p. 37Z386.

L'Aurignacien du plateau de Belcayre (Dordogne'), Périgueux, 1934, p. 379-38т, ill. Gravure aurignacienne de Belcayre (I)ordogn'), Périgueux, 1934, p. 388-392, ill. L'Abri des Merveilles ì C'astelmerle (Sergeac, Dordogne), Toulouse, 1936, p. 5i8608 , ill.

GALLIA:

Fouilles effectuees au "Chateau des lars" (commune de Saint-Mlerd-les-Oussines) (en coll. avec L. Prieur), V, 1, 1947, p. 47-79, pl.. ill.

NOS PAYS DU CENTRE-OUEST:

Au royaume des troglodytes: la grotte de Lascaux, 1947, p. 8-11, ill.

REVUE ANTHROPOLOGIQUE:

Le Moustérien de Belcayre: notes de morphologie, XXXVII, 1927, 12 p., 3 pl.

REVUE NUMISMATIQUE:

Le trésor de Vaulry et les monnaies gauloises du Limousin, 1937, p. 52-70, ill.

MELANGES BEGOUEN:

La grotte de la Balutie, 'T'oulouse, Privat, 1939, p. 161-169, ill.

A paraître:

Statuette gauloise à Chateau-Chervix (Haute-Vienne) (Bull. Soc. archéol. et hist. du Limousin, LXXXIV, 1951).

Les arts en Limousin ì l'époque (ibid.).

La villa gallo-romaine d'Antone à Pierre-Buffière (Haute-Vienne).] 